\title{
Our commitment is to patient partnership
}

\author{
Fiona Godlee editor in chief
}

The BMJ

Evidence that leads to changes practice is rarer than we might hope. In partnership with the MAGIC non-profit research and innovation programme (http://magicproject.org), we are now on the lookout for such practice changing evidence on which to base our "Rapid Recommendations" series. So far we have published one on whether patients with severe symptomatic aortic stenosis who are at low to intermediate surgical risk should have transcatheter or surgical valve replacement (doi:10. 1136/bmj.i5085). We will soon be looking at knee arthroscopy and then drug treatments to reduce maternal transmission of HIV to the fetus.

This week we focus on low intensity pulsed ultrasound (LIPUS), a form of bone stimulation intended to promote healing. As recounted by Rudolf Poolman and colleagues (doi:10.1136/bmj. j576), LIPUS was approved in the United States in 1994 for fracture healing and in 2000 for treatment of non-union. In 2010 the National Institute for Care and Health Excellence approved it for similar indications in the United Kingdom. LIPUS is now widely used in the developed world. But does it work?

The Rapid Recommendations panel of non-conflicted reviewers has concluded that it doesn't. Prompted by the TRUST trial, published in The BMJ last year (doi:10.1136/bmj.i5351), they collaborated with others on a systematic review (doi:10.1136/ bmj.j656), incorporating 26 randomised trials. The trials at lowest risk of bias consistently found no difference between
LIPUS and sham or no ultrasound. On the basis of this evidence, and the costly and cumbersome devices required, the panel makes a strong recommendation, with moderate to high certainty, against using LIPUS for bone healing.

Crucially, the key outcomes were those that are important to patients. So, instead of simply looking at time to radiographic bone healing, the review focused on time to return to work, time to full weight bearing, and the number of subsequent operations.

The emphasis on these outcomes is a good sign of progress towards more patient centred research. But there is much still to do. When we ask authors to tell us how patients were involved in their research, the answer is almost always not at all. We now publish statements on patient involvement in every research article to encourage a change in the culture. Other elements of our patient partnership initiative (bmj.com/campaign/patientpartnership) include review by patients of research articles, patient co-creation of educational articles, and patients' involvement in The BMJ's events. For the past three years we have been lucky enough to work with our inspirational patient editor, Rosamund Snow. Her death earlier this month is a great blow to us and all who knew her (doi:10.1136/bmj.j850; http:/ /blogs.bmj.com/bmj/2017/02/15/paul-buchanan-on-rosamundsnow). But with the ongoing help of our patient panel we are more determined than ever to continue our advocacy for patient partnership in healthcare. 